\title{
Combining inhibitor tolerance and D-xylose fermentation in industrial Saccharomyces cerevisiae for efficient lignocellulose-based bioethanol production
}

Mekonnen M Demeke ${ }^{1,2}$, Françoise Dumortier ${ }^{1,2}$, Yingying Li ${ }^{1,2}$, Tom Broeckx ${ }^{1,2}$, María R Foulquié-Moreno ${ }^{1,2}$ and Johan M Thevelein ${ }^{1,2^{*}}$

\begin{abstract}
Background: In addition to efficient pentose utilization, high inhibitor tolerance is a key trait required in any organism used for economically viable industrial bioethanol production with lignocellulose biomass. Although recent work has succeeded in establishing efficient xylose fermentation in robust industrial Saccharomyces cerevisiae strains, the resulting strains still lacked sufficient inhibitor tolerance for efficient sugar fermentation in lignocellulose hydrolysates. The aim of the present work was to combine high xylose fermentation activity and high inhibitor tolerance in a single industrial yeast strain.

Results: We have screened 580 yeast strains for high inhibitor tolerance using undetoxified acid-pretreated spruce hydrolysate and identified a triploid industrial baker's yeast strain as having the highest inhibitor tolerance. From this strain, a mating competent diploid segregant with even higher inhibitor tolerance was obtained. It was crossed with the recently developed D-xylose fermenting diploid industrial strain GS1.11-26, with the Ethanol Red genetic background. Screening of 819 diploid segregants from the tetraploid hybrid resulted in two strains, GSF335 and GSF767, combining high inhibitor tolerance and efficient xylose fermentation. In a parallel approach, meiotic recombination of GS1.11-26 with a haploid segregant of Ethanol Red and screening of 104 segregants resulted in a similar inhibitor tolerant diploid strain, GSE16. The three superior strains exhibited significantly improved tolerance to inhibitors in spruce hydrolysate, higher glucose consumption rates, higher aerobic growth rates and higher maximal ethanol accumulation capacity in very-high gravity fermentation, compared to GS1.11-26. In complex medium, the D-xylose utilization rate by the three superior strains ranged from 0.36 to $0.67 \mathrm{~g} / \mathrm{g}$ DW/h, which was lower than that of GS1.11-26 (1.10 g/g DW/h). On the other hand, in batch fermentation of undetoxified acid-pretreated spruce hydrolysate, the three superior strains showed comparable D-xylose utilization rates as GS1.11-26, probably because of their higher inhibitor tolerance. They produced up to $23 \%$ more ethanol compared to Ethanol Red.
\end{abstract}

Conclusions: We have successfully constructed three superior industrial S. cerevisiae strains that combine efficient D-xylose utilization with high inhibitor tolerance. Since the background strain Ethanol Red has a proven record of successful industrial application, the three new superior strains have strong potential for direct application in industrial bioethanol production.

Keywords: Bioethanol production, Pentose utilization, Inhibitor tolerance, Saccharomyces cerevisiae, Meiotic recombination, Spruce hydrolysate, Very-high gravity fermentation

\footnotetext{
* Correspondence: johan.thevelein@mmbio.vib-kuleuven.be

'Laboratory of Molecular Cell Biology, Institute of Botany and Microbiology,

KU Leuven, Leuven-Heverlee, Flanders B-3001, Belgium

${ }^{2}$ Department of Molecular Microbiology, VIB, Kasteelpark Arenberg 31,

Leuven-Heverlee, Flanders B-3001, Belgium
} 


\section{Background}

Biofuels produced from non-food lignocellulosic biomass, such as agricultural and forest residues, municipal solid wastes and energy crops are believed to be an important sustainable solution for the future transport energy deficit and the green house gas emission problem [1]. Such lignocellulosic materials constitute the most abundant organic materials in the biosphere and thus represent a huge and renewable reservoir for transport energy [2]. Although important technological advances have been realized to exploit this potential in the last three decades, so-called second-generation biofuel production is not yet feasible in an economically viable way $[3,4]$.

Bio-ethanol is currently the dominant renewable biofuel used in the transportation sector [5]. It has already been introduced on a large scale in various countries, such as Brazil, the US, and increasingly in European countries, and is now predominantly produced from food crops. In the past few years, substantial efforts have been focused on production of bioethanol from non-food lignocellulose biomass. Bioethanol production from lignocellulosic wastes, such as crop residues and sugar cane bagasse, and from cultivation of bioenergy crops, has the potential to contribute significantly to the replacement of fossil fuel for transportation purposes [6,7].

The main challenges in advanced bioethanol production are the development of efficient and cheap technologies to liberate all fermentable sugars from lignocellulosic feedstocks, and the engineering of robust microorganisms able to rapidly ferment all sugar present in the biomass hydrolysate, mainly glucose and D-xylose [8,9]. Several pretreatment and enzymatic hydrolysis processes have been reported with increasing efficiency for releasing the sugars from the biomass [10-12]. Yeast strains have even been developed that secrete cellulolytic enzymes for use in consolidated bioprocessing [13]. However, in addition to the release of fermentable sugars, large amounts of several types of inhibitory compounds are released during the pretreatment process. They inhibit microbial fermentation and growth, resulting in severely reduced ethanol yield and productivity. Therefore, economically viable industrial production of lignocellulose-derived bioethanol requires not only a microorganism that is able to ferment all hexose and pentose monosaccharides in the lignocellulose hydrolysates, but also exhibits unusually high tolerance to the toxic compounds present in the lignocellulose hydrolysates.

Substantial progress has been made in the past few years to develop yeast strains that are able to ferment D-xylose [14-19], and to obtain strains with improved inhibitor tolerance [20-22]. Some D-xylose fermenting recombinant strains of $S$. cerevisiae and natural D-xylose utilizing yeast species with improved inhibitor tolerance have also been reported [23,24]. However, most of this work has been performed with laboratory $S$. cerevisiae strains or strains of $S$. cerevisiae and other yeast species without proven track record in industrial bioethanol production. In addition, the performance of the best strains available in terms of D-xylose fermentation and inhibitor tolerance still requires much improvement in order to reach efficient fermentation of lignocellulosic hydrolysates, especially at a higher solid loading [25]. Since pentose fermentation appears to be much more sensitive to the toxic inhibitors [26], the productivity of the yeast in high-density lignocellulose hydrolysates is largely determined by the robustness of the pentose fermentation.

Recently, a D-xylose fermenting strain GS1.11-26 has been developed from Ethanol Red, a prime industrial yeast strain used in first-generation bioethanol production with corn and wheat [27]. Ethanol Red has a proven track record of excellent fermentation capacity and yield, high robustness and stress tolerance, excellent performance in fed-batch production on molasses, tolerance to dehydration and maintenance of high vitality during storage and transport. For that reason, the strain GS1.11-26 was considered to have very promising potential for development of an all-round robust yeast strain for efficient fermentation of various lignocellulosic materials. However, due to the accumulation of background mutations during the mutagenesis and/or evolutionary engineering procedures used to develop the strain, GS1.11-26 did not retain the same tolerance to high concentrations of ethanol and acetate, and showed reduced ethanol accumulation capacity in very high-density fermentations compared to the original Ethanol Red strain. Moreover, it also had a partial respiratory defect causing a reduced aerobic growth rate, which would compromise large-scale propagation of yeast in fed-batch mode [27]. Hence, as such the strain would not be suitable for direct industrial application.

We now report the development of three new xyloseutilizing industrial yeast strains, derived from the GS1.11-26 strain, and which lack its negative properties. The new strains are diploid and were obtained through meiotic recombination with a diploid segregant from a strongly inhibitor-tolerant triploid strain and with a haploid segregant of Ethanol Red. The first strain was the most inhibitor tolerant strain identified by screening a large collection of yeast strains for tolerance to undetoxified acid-pretreated spruce hydrolysate. The three new superior strains exhibited significantly increased tolerance to various inhibitors in spruce hydrolysate, faster growth rate in glucose medium and a faster glucose consumption rate and higher ethanol accumulation capacity in very high gravity fermentations. The maximum D-xylose utilization rate of the three new strains was slower than that of GS1.11-26, but they completely consumed $37 \mathrm{~g} / \mathrm{L}$ D-xylose and $36 \mathrm{~g} / \mathrm{L}$ glucose in about $32 \mathrm{~h}$. Our results also demonstrate that commercially important traits present in diploid 
industrial yeast strains can be combined into a single industrial yeast strain with superior properties and performance without the need for isolation of haploid derivatives.

\section{Results}

Screening of S. cerevisiae strain collection for tolerance to inhibitors in spruce hydrolysate

We first aimed at obtaining a strain with extremely high performance in terms of growth and fermentation directly in inhibitor-rich lignocellulose hydrolysate, since simultaneous tolerance to multiple inhibitors is important for high productivity in lignocellulose hydrolysates [28]. For that purpose, undetoxified acid-pretreated spruce hydrolysate was used as medium for screening, because this hydrolysate contains more inhibitors and in higher concentrations than most other hydrolysates $[29,30]$. Using this medium, we screened 580 different $S$. cerevisiae strains first for the ability to grow in different concentrations of spruce hydrolysate. The strain collection consisted of laboratory strains, industrial strains (wine, beer, baker's and bioethanol production strains) as well as a variety of natural isolates from various sources. The first screening was done with growth tests on YP-agar plates containing up to $70 \%$ whole slurry of the spruce hydrolysate at $\mathrm{pH} 5.5$.

From the 580 strains tested, 35 strains that performed the best in the plate test were subsequently screened in more detail by determining their fermentation performance in $30 \%$ spruce hydrolysate supplemented with YP and glucose to $200 \mathrm{~g} / \mathrm{L}$, at $\mathrm{pH}$ 5.5. The high concentration of glucose was used to obtain high osmotic stress at the beginning and high ethanol stress at the end of the fermentation. The industrial strain Ethanol Red was used as control. Out of the 35 strains tested, we selected 14 strains that showed at least similar fermentation performance as the industrial strain Ethanol Red. Finally, the fermentation performance of the 14 best strains was tested again in a single fermentation experiment and ten strains were found to perform consistently better than Ethanol Red. They mainly showed a shorter lag phase (Figure 1). The best strain, JT21653, which showed both a shorter lag phase and a more complete attenuation of the sugar, was chosen for further analysis. JT21653 is a baker's yeast that was purchased from a local commercial source. A species identification test was performed on a single cell isolate at the BCCM/MUCL (Mycothèque de l'Université Catholique de Louvain, Louvain-la-Neuve, Belgium), which confirmed that the yeast was $S$. cerevisiae Meyen ex. E.C. Hansen. Flow cytometry analysis of the DNA content revealed that JT21653 had a triploid genome (Figure 2). The strain showed good sporulation and high spore viability and hence, was selected for further analysis.

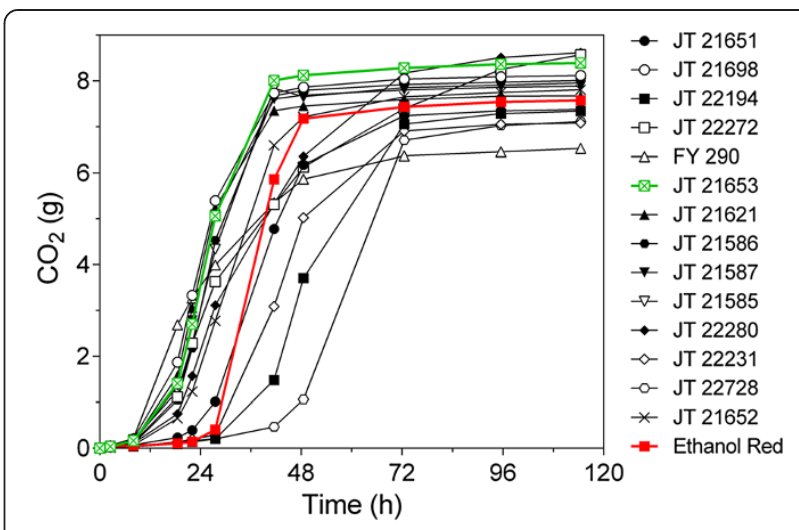

Figure 1 Fermentation performance of the 14 different preselected inhibitor tolerant yeast strains in comparison to Ethanol Red. Semi-anaerobic fermentation was performed in 30\% pretreated slurry of spruce hydrolysate supplemented with YP and glucose to $200 \mathrm{~g} / \mathrm{L}$ at pH 5.5. The course of the fermentation was followed by weight loss due to $\mathrm{CO}_{2}$ production during the fermentation. The robust industrial strain Ethanol Red (red) was used as a reference, strain JT21653 (green) was selected as the best performing strain.

Isolation of an inhibitor tolerant segregant of JT21653

In order to select a segregant of JT21653 with an inhibitor tolerance at least as good as the triploid parent, we screened 41 segregants of JT21653 for fermentation performance under semi-anaerobic conditions in $40 \%$ spruce hydrolysate supplemented with YP and glucose to $200 \mathrm{~g} / \mathrm{L}$ at $\mathrm{pH} 5.5$ (results not shown). From this prescreening, we selected the 15 segregants with the best fermentation performance. For selection of the most inhibitor-tolerant segregant, the concentration of the spruce hydrolysate was increased to $77 \%$ and supplemented with $70 \mathrm{~g} / \mathrm{L}$ glucose. This concentration of spruce hydrolysate was severely inhibitory to the industrial strain Ethanol Red, the triploid parent JT21653 as well as the majority of the 15 segregants, whereas four segregants were able to complete the fermentation under these conditions albeit with varying rates (Figure 3 ). One segregant, named Fseg25, performed exceptionally well, initiating the fermentation with virtually no lag phase and completing it already after about $48 \mathrm{~h}$, a time at which only other segregant had made a significant start-up of the fermentation. Analysis of the DNA content of Fseg25 by flow cytometry indicated that the strain had a diploid genome (Figure 2). In addition, it was able to mate with a $M A T \alpha / \alpha$ strain, indicating that it was MATa/a and the resulting tetraploid strain produced viable spores. Therefore, the Fseg25 segregant was chosen as a mating partner for genetic recombination with the diploid $M A T \alpha / \alpha$ strain GS1.11-26.

\section{Meiotic recombination of GS1.11-26 with Fseg25}

To combine the superior D-xylose fermentation performance of GS1.11-26 with the superior inhibitor tolerance trait of Fseg25, we mated the MAT $\alpha / \alpha$ strain GS1.11-26 

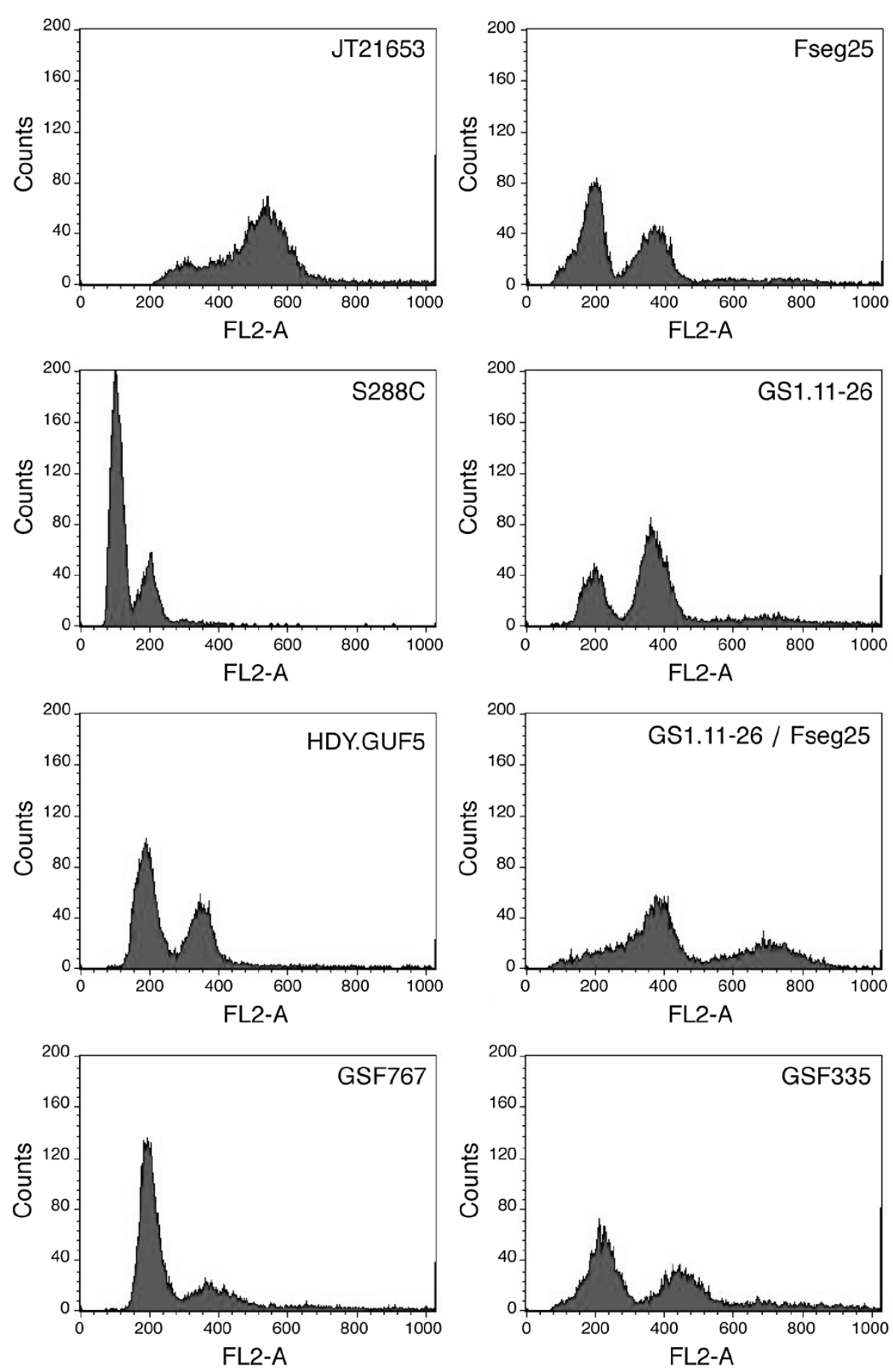

Figure 2 Determination of DNA content by flow cytometry. Strains were grown to exponential phase, after which they were fixed with ethanol, and the DNA was stained with propidium iodide. Control haploid (S288c) and diploid (HDY.GUF5) strains were used for comparison.

with the MATa/a segregant Fseg25. The tetraploid strain generated was sporulated, and 819 meiotic segregants were screened in order to select diploid hybrid F1 segregants with the required properties, mainly efficient D-xylose fermentation, fast aerobic growth and high inhibitor tolerance in spruce hydrolysate.

\section{Screening for D-xylose fermentation capacity}

We first performed a prescreening of the 819 segregants based on their ability to grow on YPX solid medium, in order to reduce the number of strains to be evaluated in fermentations. All segregants that showed detectable growth on solid xylose medium were further analyzed 


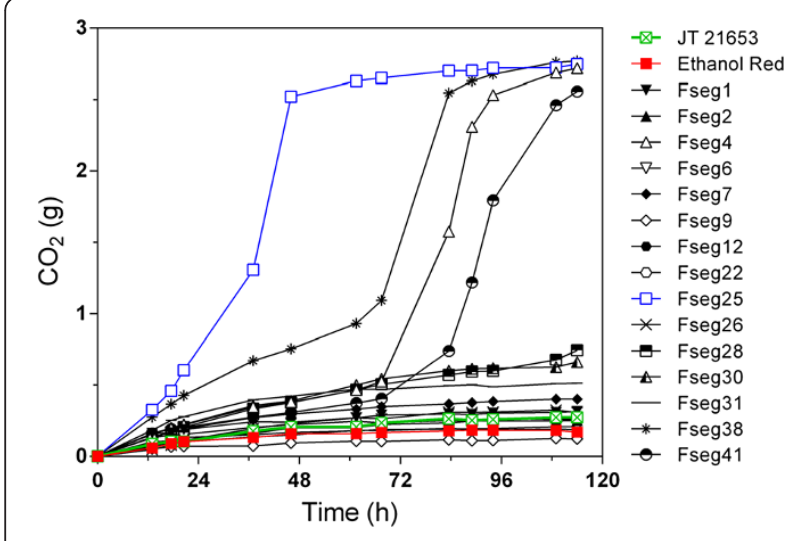

Figure 3 Selection of a superior segregant from the inhibitor tolerant baker's yeast JT21653. Fermentation was performed in semi-anaerobic conditions using treated slurry of spruce material at $77 \%$ concentration and supplemented with $70 \mathrm{~g} / \mathrm{L}$ glucose. $\mathrm{CO}_{2}$ production was estimated from the weight loss. Fseg25 (blue) showed the best performance, with in particular a very short lag phase compared to the other segregants. The diploid parent JT21653 (green) and the industrial bioethanol production strain Ethanol Red (red) as well as all other segregants, except for four, were not able to ferment appreciably at this concentration of spruce hydrolysate within $120 \mathrm{~h}$.

for growth in liquid medium. For that purpose, the strains were inoculated in $1 \mathrm{~mL}$ YPX medium at an initial $\mathrm{OD}_{600}$ of 1.0. After about $24 \mathrm{~h}$ incubation, a range of cell densities, from $\mathrm{OD}_{600}$ of about 5 up to 33 was observed for the different segregants. Strain GS1.11-26 showed an $\mathrm{OD}_{600}$ between 28 and 33 in different replicate growth assays. To monitor the correlation between growth in liquid YPX and fermentation performance, segregants growing to an $\mathrm{OD}_{600}$ above 5 were evaluated by fermentation in YP medium containing $40 \mathrm{~g} / \mathrm{L} \mathrm{D}$-xylose. We observed that, most of the best D-xylose fermenting strains also performed well in such growth evaluation experiments (data not shown). Thus, the majority of poor D-xylose fermenting segregants could be excluded by using a cut-off value for growth to an $\mathrm{OD}_{600}$ of 15 in $24 \mathrm{~h}$, since all the good D-xylose fermenting segregants grew to an $\mathrm{OD}_{600}$ of above 15. Hence, growth in liquid YPX medium for $24 \mathrm{~h}$ and selection of the segregants growing to a minimum $\mathrm{OD}_{600}$ of 15 was considered to be the best method for rapid initial screening and elimination of poor performers.

Using this method, about 168 segregants growing to $\mathrm{OD}_{600}$ values of about 15 in $24 \mathrm{~h}$ were preselected and further tested for D-xylose fermentation performance in semi-anaerobic conditions. This was done in different batches of experiments (results not shown) and finally resulted in 48 segregants with moderate to rapid D-xylose fermentation capacity (Figure 4). To allow a proper comparison, the 48 selected segregants were evaluated in a single batch of fermentation experiments. The 27 best

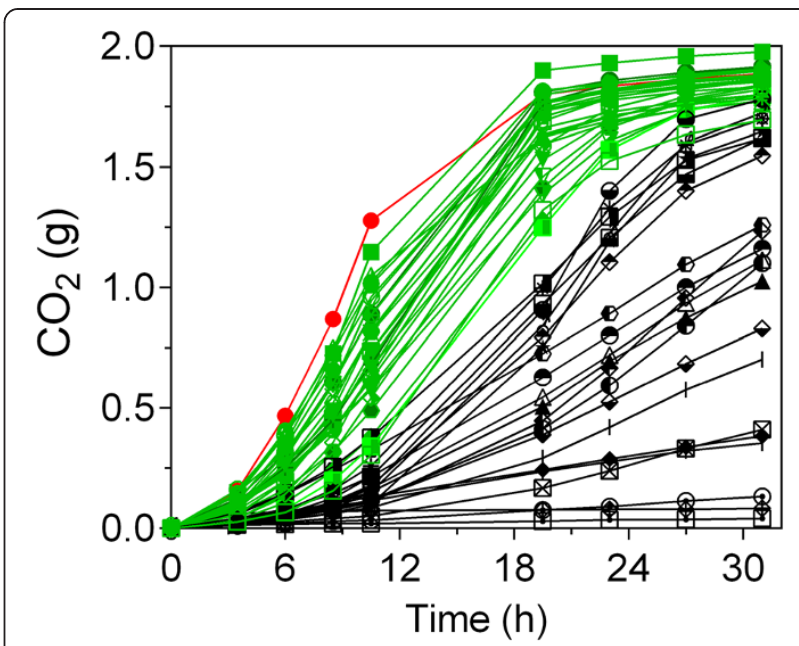

Figure 4 D-xylose fermentation profile of the 48 preselected D-xylose utilizing segregants obtained from the tetraploid strain GS1.11-26/Fseg25, in YP medium containing $40 \mathrm{~g} / \mathrm{L}$ D-xylose. The 27 selected segregants with the best performance are shown in green and the parent strain GS1.11-26 is shown in red. A similar fermentation profile was obtained during the preselection step in the same conditions.

segregants, with a D-xylose fermentation performance close to that of GS1.11-26, were eventually selected for further analysis (Figure 4). Flow cytometry analysis showed that the selected 27 segregants all had a DNA content similar to that of a diploid control strain (data not shown). Hence, all segregants appeared to be diploid strains, although aneuploidy for one or more chromosomes cannot be ruled out.

\section{Screening of hybrid segregants for aerobic growth rate and inhibitor tolerance}

One of the shortcomings of GS1.11-26 for direct industrial application was the slow aerobic growth rate, which is a key factor for yeast propagation in the preparation of sufficient inoculum to start the industrial fermentation process. Hence, we screened the $27 \mathrm{D}$-xylose fermenting strains for growth rate in synthetic medium containing glucose as a carbon source. The recombinant strain HDY.GUF5 (Ethanol Red background) was used as a reference throughout this work since GS1.11-26 was developed from this strain background [27]. From the 27 segregants, only six showed a growth rate as high as that of the HDY. GUF5 strain (Figure 5). When these six segregants were evaluated in inhibitor-rich spruce hydrolysate, only two segregants, named GSF335 and GSF767, grew better than the HDY.GUF5 strain (data not shown). Both strains were found to be diploid and had $M A T \alpha / \alpha$ mating type. They were selected for further evaluation. 


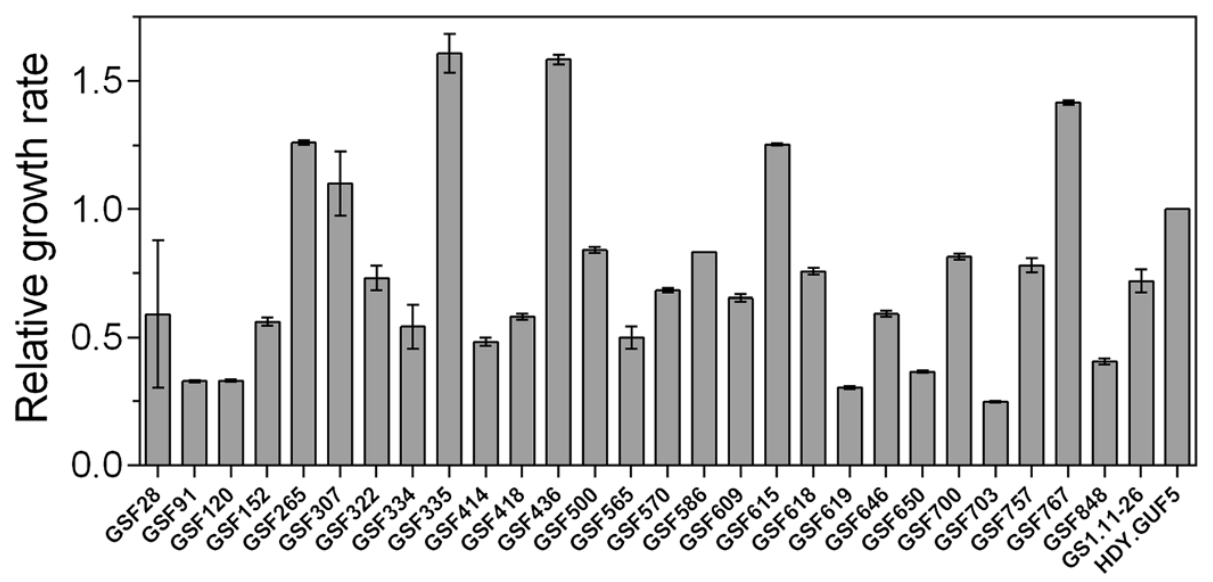

Figure 5 Relative maximum growth rate of the 27 best D-xylose fermenting segregants in comparison to the strain HDY.GUF5. Growth was performed in synthetic medium containing $20 \mathrm{~g} / \mathrm{L}$ glucose in $200 \mu \mathrm{l}$ volume using a bioscreen assay at an initial $\mathrm{OD}_{600}$ of 0.1 . Error bars represent standard deviation from average values of triplicate experiments. The maximum growth rate obtained for each strain was calculated relative to HDY.GUF5.

\section{Backcrossing the diploid GS1.11-26 with a haploid} segregant of Ethanol Red

In a parallel approach, the $M A T \alpha / \alpha$ diploid strain, GS1.11-26, with high D-xylose fermentation activity, was crossed with the segregant ER17 of Ethanol Red. ER17 was previously selected among a number of segregants for its higher acetic acid tolerance in fermentation, which was even better than that of its diploid parent strain Ethanol Red (Meijnen et al., unpublished data).

The crossing of GS1.11-26 with ER17 resulted in a triploid strain. Meiotic segregation in triploid strains often produces two haploid and two diploid progeny in a tetrad [31]. Obtaining diploid progeny is important since haploid strains are unattractive for industrial application, because of their lower genetic stability and robustness [32]. The triploid strain was sporulated and 104 segregants were isolated. Most of the tetrads produced four viable spores. All the isolates were then evaluated for the important phenotypic traits.

\section{Screening of the hybrid segregants from GS1.11-26/ER17 for growth in D-xylose medium}

The 104 segregants isolated from GS1.11-26/ER17 were first screened for growth in YPX medium as a preselection step. A range of final $\mathrm{OD}_{600}$ values from about 2 to 33 was observed (Figure 6), indicating the involvement of multiple genetic factors for D-xylose growth. Since growth on and fermentation of D-xylose have previously shown a good correlation, the 21 segregants that grew best in D-xylose medium were selected for the next evaluation.

\section{Screening of the hybrid segregants from GS1.11-26/ER17 for aerobic growth rate}

The 21 best $\mathrm{D}$-xylose growing segregants were further tested for growth rate in glucose medium. They were pregrown in $3 \mathrm{~mL}$ YPD medium. Segregants that yielded after $16 \mathrm{~h}$ much lower biomass than HDY.GUF5 were excluded. The remaining seven segregants were tested for growth rate in synthetic medium containing $20 \mathrm{~g} / \mathrm{L}$ glucose. Two segregants that produced after $16 \mathrm{~h}$ lower $\mathrm{OD}_{600}$ values than HDY.GUF5 were included for comparison.

In the growth rate assay, seven of the nine strains tested showed a much faster growth rate than GS1.11-26 (Figure 7). The two segregants, GSE17 and GSE102, that produced lower $\mathrm{OD}_{600}$ values during the preselection step, also showed a slower growth rate than GS1.11-26, confirming the results from the pregrowth assay. The seven segregants that grew with a rate close to that of HDY.GUF5 were then selected for the final fermentation experiment in D-xylose medium.

\section{Selection of the most superior D-xylose fermenting and inhibitor tolerant hybrid strains}

To select first the best $\mathrm{D}$-xylose fermenting strains, the seven hybrid strains with a rapid growth rate in glucose were evaluated for fermentation performance in $50 \mathrm{~mL}$ YP medium containing $40 \mathrm{~g} / \mathrm{L}$ D-xylose as a sole carbon source. The original evolved strain GS1.11-26 was used for comparison. As can be seen in Figure 8, two strains, GSE44 and GSE16, utilized D-xylose very well, nearly as well as GS1.11-26. Their rate of D-xylose utilization (estimated from the $\mathrm{CO}_{2}$ production rate) was slightly slower than that of GS1.11-26.

These two strains, GSE44 and GSE16, were tested for tolerance to acetic acid and to spruce hydrolysate. GSE16 showed similar tolerance towards acetic acid and inhibitors in spruce hydrolysate as the HDY.GUF5 strain (growth in liquid YPD containing $6 \mathrm{~g} / \mathrm{L}$ acetic acid, $\mathrm{pH} 4.5$, or $80 \%$ of the liquid portion of spruce hydrolysate, $\mathrm{pH} 5$ ). On the other hand, GSE44 displayed reduced tolerance 


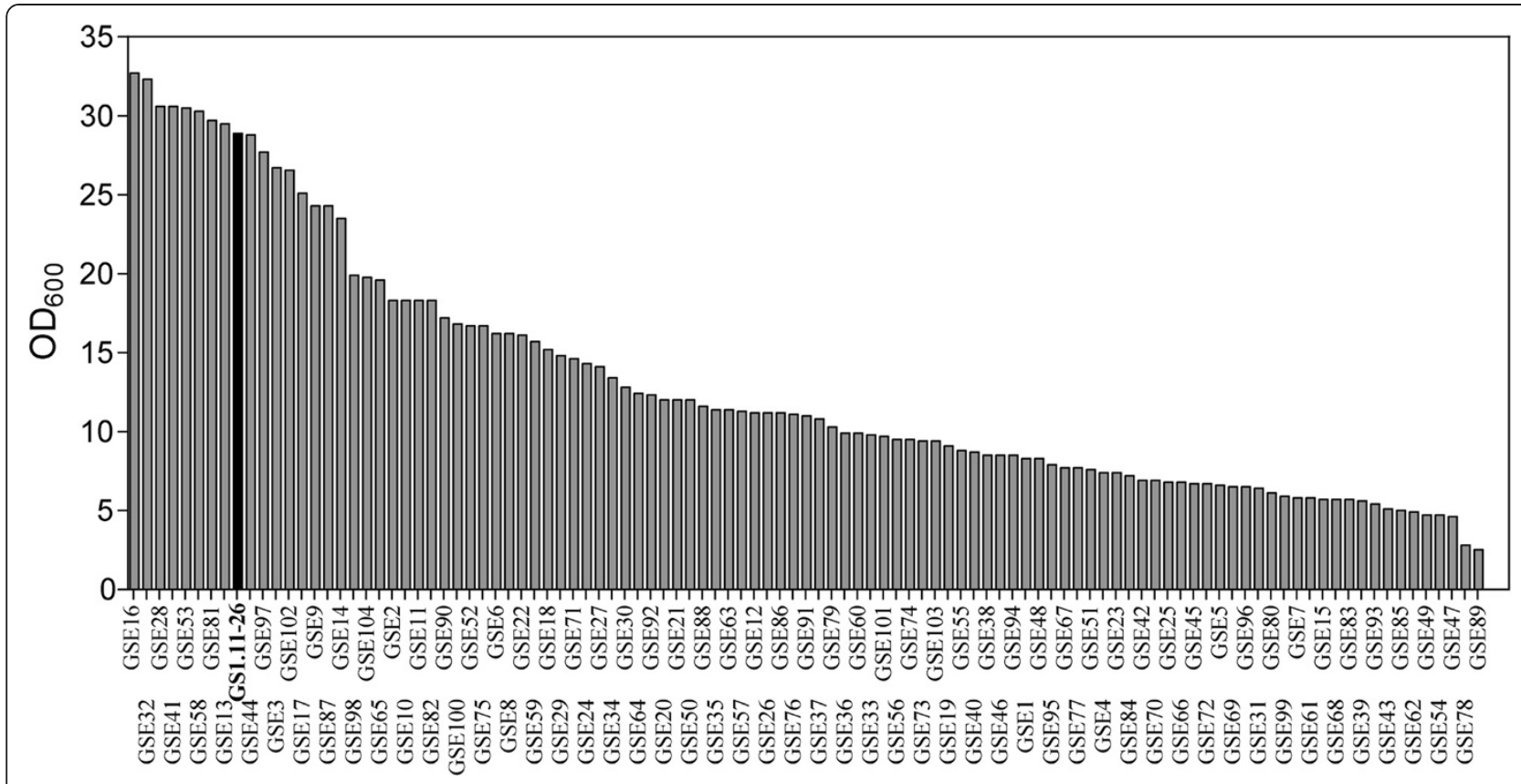

Figure 6 Screening of 104 segregants obtained from the triploid strain GS1.11-26/ER17, for growth in $1 \mathrm{~mL}$ YP medium containing $20 \mathrm{~g} / \mathrm{L}$ D-xylose as carbon source. Cells were inoculated at an $\mathrm{OD}_{600}$ value of 1.0. The final $\mathrm{OD}_{600}$ was then measured after $20 \mathrm{~h}$. The parent strain GS1.11-26 was used as a reference.

especially to acetic acid, showing growth in the presence of only up to $4 \mathrm{~g} / \mathrm{L}$ acetic acid and no growth anymore at $5 \mathrm{~g} / \mathrm{L}$ acetic acid (results not shown). Hence, the diploid strain GSE16, which is $M A T a / \alpha$, was selected for further evaluation.

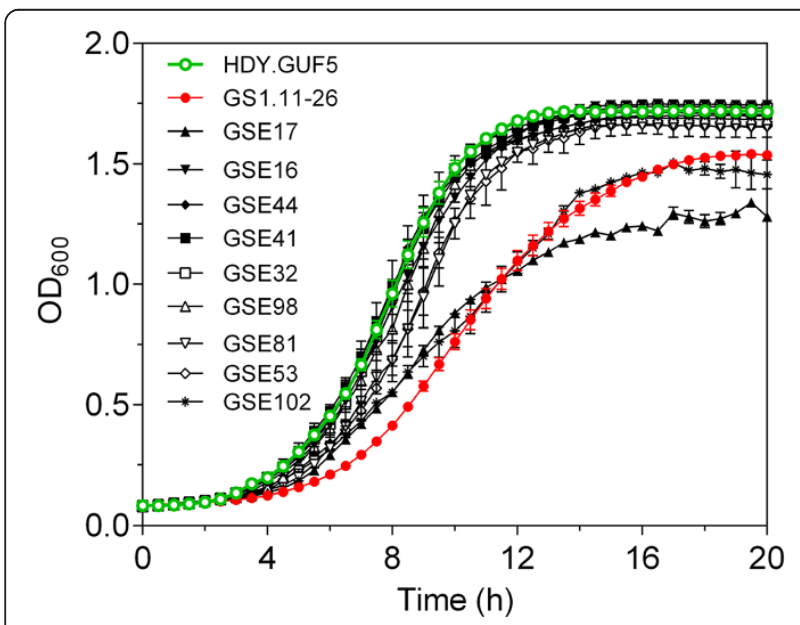

Figure 7 Screening of the best D-xylose growing segregants, obtained from the triploid strain GS1.11-26/ER17, for aerobic growth rate in glucose medium. A bioscreen assay was performed in $200 \mu \mathrm{l}$ volume synthetic medium containing $20 \mathrm{~g} / \mathrm{L}$ glucose, at an initial $\mathrm{OD}_{600}$ of 0.1. Error bars represent standard deviation from the average in triplicate experiments.

\section{Evaluation of the most superior hybrid strains} Fermentation performance with a glucose-D-xylose mixture The three selected strains, GSE16 (obtained from backcrossing GS1.11-26 with a segregant of Ethanol Red), GSF335 and GSF767 (obtained from crossing GS1.11-26 with a segregant of JT21653), were evaluated in a more controlled fermentation experiment in $100 \mathrm{~mL}$ YP medium containing $36 \mathrm{~g} / \mathrm{L}$ glucose and $37 \mathrm{~g} / \mathrm{L}$ D-xylose at $35^{\circ} \mathrm{C}$. The inoculum cell density was $1.3 \mathrm{~g} \mathrm{DW} / \mathrm{L}$. The

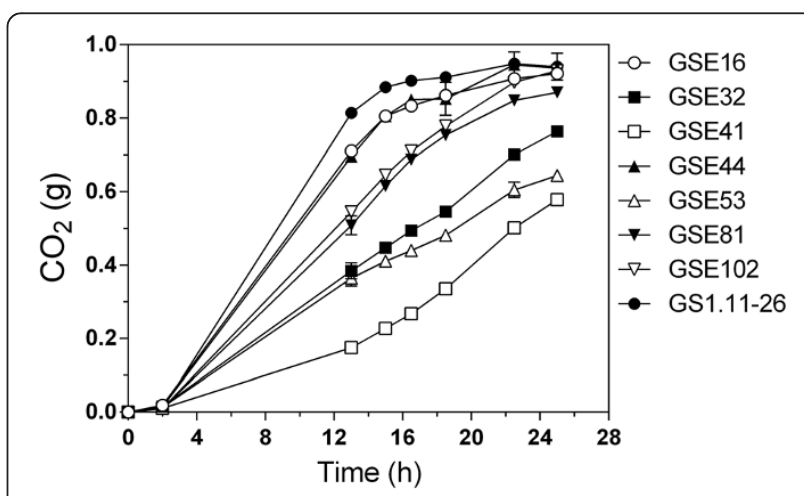

Figure 8 Selection of efficient $\mathrm{D}$-xylose fermenting segregants from the seven segregants with best performance, obtained from the triploid strain GS1.11-26/ER17. The fermentations were performed in $50 \mathrm{~mL}$ volume with YP medium containing $40 \mathrm{~g} / \mathrm{L}$ D-xylose. The $\mathrm{CO}_{2}$ release was estimated from the weight loss during fermentation. Error bars represent standard deviation from the average in duplicate experiments. 

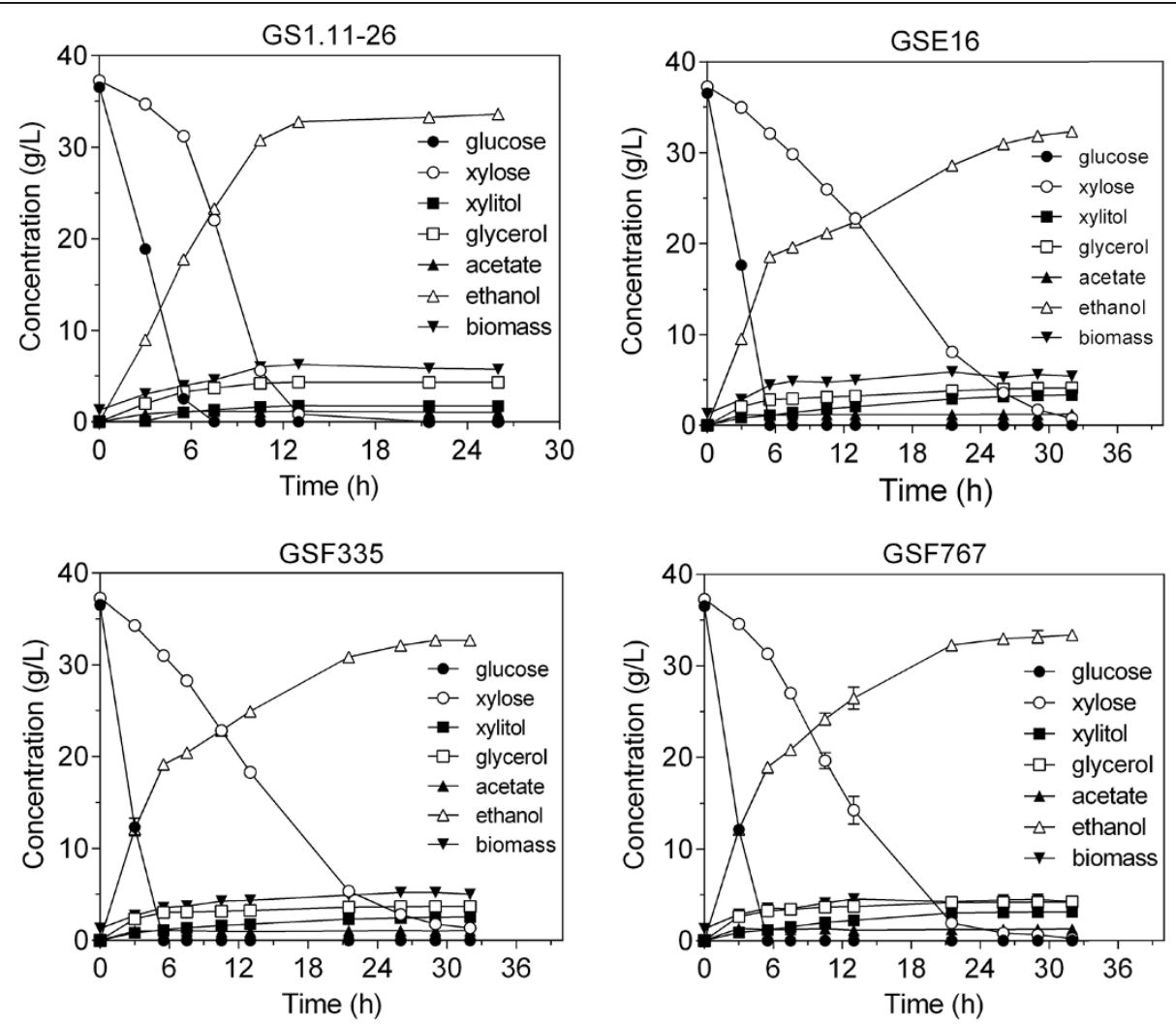

Figure 9 Fermentation performance of the three new hybrid strains in glucose and D-xylose mixture in comparison to that of GS1.11-26. The fermentations were performed in YP medium containing $37 \mathrm{~g} / \mathrm{L}$ glucose and $37 \mathrm{~g} / \mathrm{L}$ D-xylose, with an initial biomass of $1.3 \mathrm{~g} / \mathrm{L}$. Error bars represent standard deviation from the average in duplicate experiments.

performance of the three hybrid strains was compared with that previously reported for the strain GS1.11-26. GS1.11-26 completely consumed both D-xylose and glucose in about $13 \mathrm{~h}$, compared to about $32 \mathrm{~h}$ for the other three strains [27] (Figure 9).

The glucose consumption rate with the three new hybrid strains was higher than that of the evolved strain GS1.11-26 (Figures 9 and 10a). Moreover, GSF335 showed even a slightly higher glucose consumption rate than the original parent strain HDY.GUF5 (Figure 10a). However, GS1.11-26 remained superior in terms of D-xylose consumption rate and ethanol productivity from D-xylose (Figures 9 and 10b). Among the three new hybrid strains, GSF767 showed the highest D-xylose consumption rate $(0.65 \mathrm{~g} / \mathrm{g} \mathrm{DW} / \mathrm{h})$, but its maximum D-xylose utilization rate was still about $40 \%$ lower than that of GS1.11-26 (1.10 g/g DW/h). On the other hand, the same ethanol yield was obtained with GS1.11-26 and
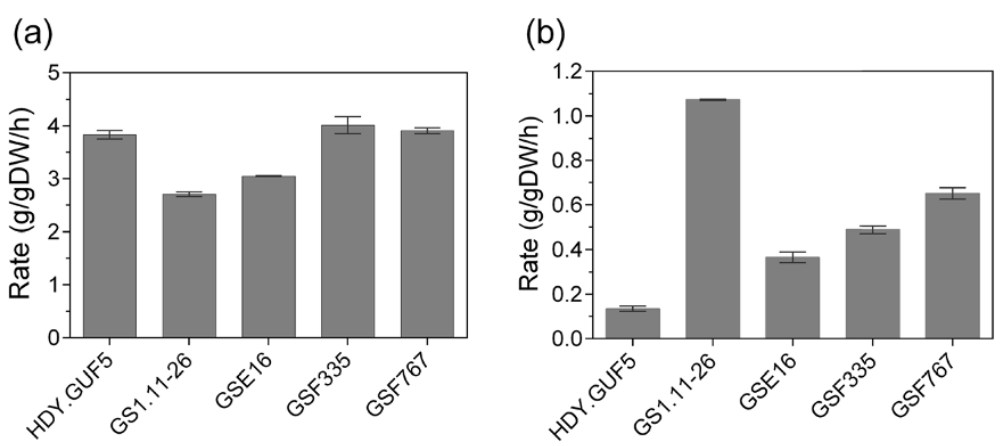

Figure 10 Maximum sugar consumption rates attained by the three new hybrid strains and GS1.11-26. (a), maximum glucose consumption rate; (b), maximum D-xylose consumption rate. The values were calculated from the fermentation experiment shown in Figure 9 . Maximum D-xylose utilization rate was obtained after glucose exhaustion. Error bars represent standard deviation from the average in duplicate experiments. 
GSF767 (0.46 g/g initial sugar or $90.2 \%$ of the theoretical maximum), while it was slightly lower with the other two strains GSF335 and GSE16 (0.44 g/g initial sugar or $86.3 \%$ of the theoretical maximum). Partial co-utilization of D-xylose and glucose was observed with all three new hybrid strains as well as with GS1.11-26, as previously reported [27] (Figure 9).

\section{Fermentation performance in inhibitor-rich spruce hydrolysate}

The three new hybrid strains, GSE16, GSF335 and GSF767, were evaluated for inhibitor tolerance in a fermentation experiment with acid pretreated spruce material. The whole slurry of the spruce material, $60 \%$, supplemented with yeast extract and peptone, was used. Glucose $(40 \mathrm{~g} / \mathrm{L})$ was added since the sugar concentration in the hydrolysate was rather low (about $13 \mathrm{~g} / \mathrm{L}$ ), which would not allow a proper comparison of the fermentation performance between the strains. Under these conditions, the rate of fermentation (as estimated from $\mathrm{CO}_{2}$ release) by GSF335 and GSE16 was much faster than that of GS1.11-26 (Figure 11). GSF767 also performed slightly better than GS1.11-26. This result indicates that the new hybrid strains, especially GSF335 and GSE16, have significantly better tolerance than GS1.11-26 towards the inhibitors present in acid pretreated spruce hydrolysate.

To evaluate more precisely the D-xylose and glucose utilization rates in the spruce hydrolysate, we studied the performance of the three strains in a similar batch fermentation using undetoxified spruce hydrolysate at a solid loading of $11 \%$ (corresponding to about $50 \%$ of the slurry), and supplemented with glucose and D-xylose to a final concentration of $62 \mathrm{~g} / \mathrm{L}$ and $18 \mathrm{~g} / \mathrm{L}$, respectively. The fermentation was performed at an initial cell density of $4 \mathrm{~g} \mathrm{DW/L}$ (Figure 12). Glucose and mannose

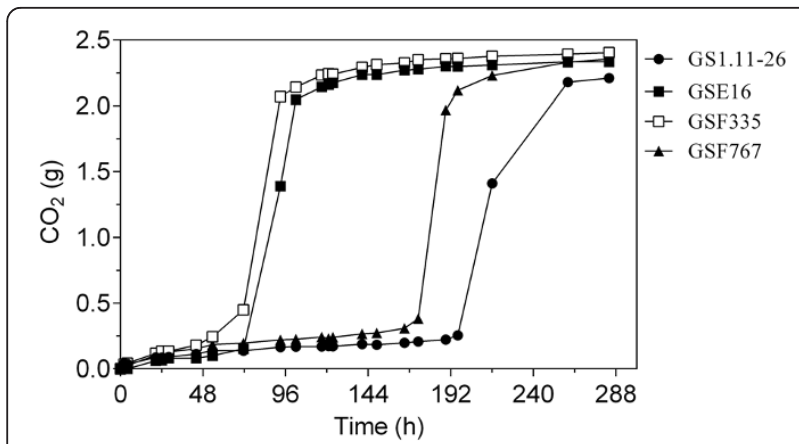

Figure 11 Inhibitor tolerance assay in acid-pretreated spruce hydrolysate with the three new hybrid strains in a small-scale fermentation experiment. The fermentation was performed using the whole slurry of spruce hydrolysate (60\%) supplemented with $40 \mathrm{~g} / \mathrm{L}$ glucose with continuous stirring at $200 \mathrm{rpm}$. Cells were inoculated at an initial cell density of $1.3 \mathrm{~g} \mathrm{DW} / \mathrm{L}$ from a stationary phase preculture in YPD medium. The $\mathrm{CO}_{2}$ production was estimated from the weight loss during fermentation. (derived from the hydrolysate) were completely consumed in less than $10 \mathrm{~h}$ by all three strains as well as by the control strains HDY.GUF5 and GS1.11-26, with GSF767 showing the fastest glucose consumption rate, using all glucose in $4 \mathrm{~h}$. The D-xylose fermentation rate was much slower compared to that of glucose fermentation and was now much more similar for all strains than in the more concentrated hydrolysate (except HDY.GUF5, which cannot use D-xylose). The hybrid strain GSF767 showed a similar D-xylose fermentation rate to that of GS1.11-26, whereas the rate was slightly lower for GSE16 and GSF335. No xylitol and little glycerol (about $0.05 \mathrm{~g} / \mathrm{g}$ ) was produced by the three hybrid strains and GS1.11-26 (results not shown). Although the D-xylose utilization rate was slower, the final ethanol concentration and yield obtained by the hybrid strains and GS1.11-26 was comparable. Compared to HDY.GUF5, the two hybrid strains GSE16 and GSF767 as well as GS1.11-26 produced about 23\% more ethanol, due to their efficient D-xylose utilization. The ethanol yield of GSF335 was lower, but still 13\% higher than that of HDY.GUF5.

\section{Fermentation performance in very-high gravity fermentation}

The hybrid strains, GSE16, GSF335 and GSF767, were also evaluated for fermentation performance in very-high gravity fermentations using YP medium containing $330 \mathrm{~g} / \mathrm{L}$ glucose. The wild type, HDY.GUF5, and the evolved strain, GS1.11-26, were included as controls. The fermentation was performed under conditions of continuous stirring at $120 \mathrm{rpm}$ and under mainly static conditions, with only $4 \mathrm{~h}$ of stirring in the beginning. Under both conditions, the three hybrid strains performed much better than GS1.11-26 (Figure 13).

Under continuous stirring, GSF335 showed the fastest rate of fermentation (Figure 13a). It reached the same final ethanol concentration as HDY.GUF5, but about $96 \mathrm{~h}$ earlier. Moreover, GSF335 also showed the highest rate of fermentation under static fermentation conditions. However, the highest final ethanol titer in static conditions was reached by GSE16 (Figure $13 \mathrm{~b}$ and $\mathrm{d}$ ). GSF767 performed similar to HDY.GUF5 for fermentation rate, but reached a somewhat lower final ethanol titer under both conditions. Overall, the three hybrid strains performed much better than GS1.11-26 under both static and continuous stirring conditions. GSF335 showed superior performance especially in the stirred fermentation conditions accumulating the highest ethanol concentration in only $72 \mathrm{~h}$. In a separate very-high gravity fermentation experiment, the final ethanol titer accumulated by GSF335 was higher than that of both the tetraploid strain GS1.11-26/Fseg25 and the diploid strain Fseg25 (results not shown). 

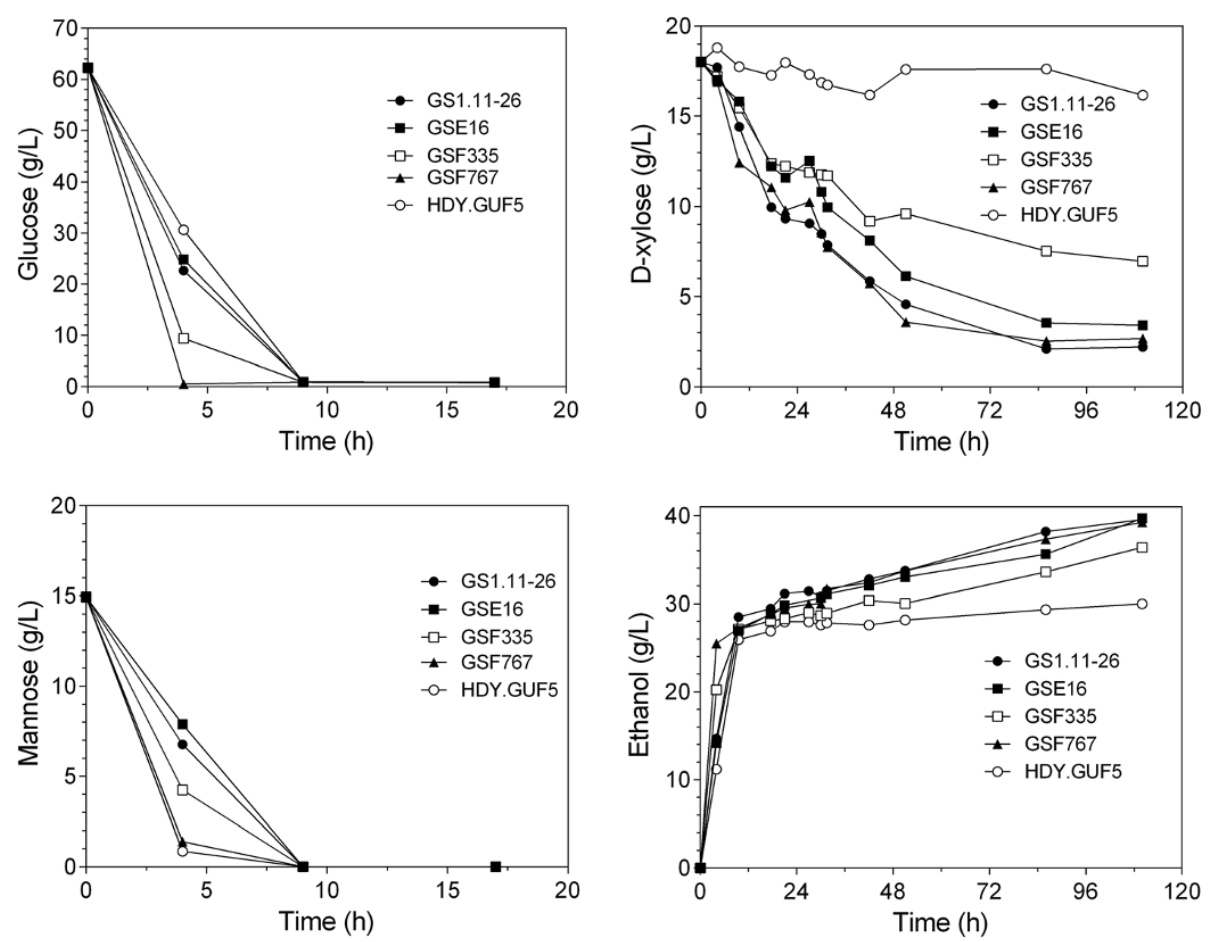

Figure 12 Fermentation profile of the three new hybrid strains in semi-anaerobic batch fermentation with acid-pretreated spruce hydrolysate. The fermentation was performed with acid-pretreated spruce hydrolysate at a solid loading of $11 \%$, supplemented with yeast nitrogen base, ammonium sulfate and amino acids. The final glucose and D-xylose concentrations were adjusted to $62 \mathrm{~g} / \mathrm{L}$ and $18 \mathrm{~g} / \mathrm{L}$, respectively. The mannose was only derived from the hydrolysate. Fermentation was started at an initial density of $4 \mathrm{~g}$ DW/L and carried out with continuous stirring at $200 \mathrm{rpm}$.

\section{Discussion}

The goal of the present paper was to develop a superior D-xylose utilizing industrial yeast strain, lacking the negative properties introduced in the background of the previously reported strain, GS1.11-26, during the mutagenesis and/or evolutionary engineering procedures used to obtain the strain. The development of the GS1.1126 strain has shown that introduction of efficient D-xylose fermentation capacity in an industrial yeast strain requires multiple genetic modifications [27]. Hence, just like stress and inhibitor tolerance, efficient D-xylose fermentation is a polygenic trait and the engineering of such traits for development of superior industrial yeast strains has been a major challenge for rational engineering strategies [33-36]. As a result, most progress in this field has been made up to now with unbiased approaches, like mutagenesis, selection and evolutionary engineering, where the underlying genetic changes responsible for the improvement in performance generally remain unknown [16,17,23,24,37-43].

In the present study, we describe the construction of three robust industrial yeast strains that combine efficient D-xylose fermentation with very high inhibitor tolerance. This was achieved by combining a number of strategies. We extended the use of classical breeding with haploid derivatives of the industrial strains to breeding with diploid strains homozygous for the mating locus. This doubles the genetic reservoir for meiotic recombination. In addition, we evaluated large arrays of segregants, using multi-step selection procedures, first aimed at eliminating the truly inferior strains with simple high-throughput tests and gradually moving the selection to conditions as close as possible to the real industrial conditions. In addition, we submitted the candidate strains to very stringent independent evaluation tests, such as the ability to accumulate a very high titer of ethanol in semi-anaerobic very-high gravity fermentations. This is a stringent quality criterion for a superior bioethanol production strain.

We have used meiotic recombination to combine the superior elements from the genomes of the D-xylose utilizing strain GS1.11-26 and the highly inhibitor tolerant strain JT21653. The latter strain was identified through screening of a collection of various yeast strains from a variety of sources using undetoxified acid pretreated spruce hydrolysate. We chose this material since it has been reported to be among the most inhibitory hydrolysates, containing multiple inhibitors, including the furan derivatives furfural and HMF, aliphatic acids, such as acetic acid, formic acid and levulinic acid, and various phenolic compounds, such as vanillic acid, vanillin, synringaldehyde, syringic acid, and 4-hydroxybenzoic acid 


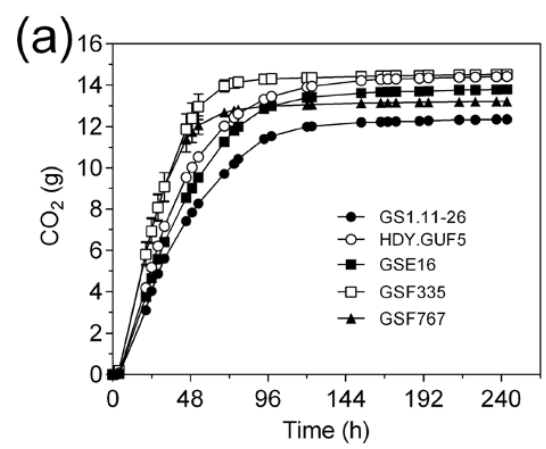

(c)

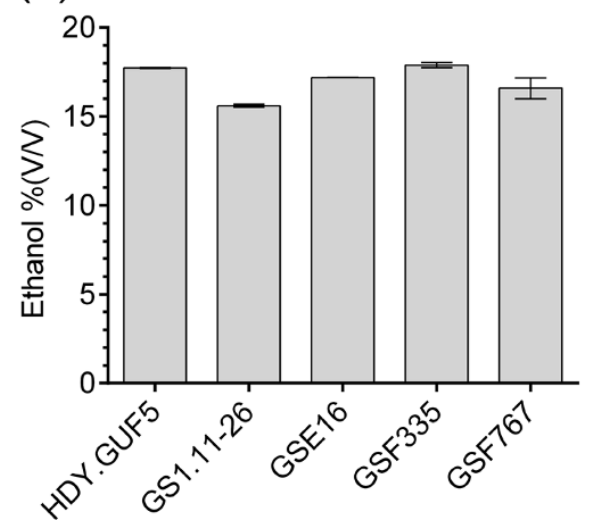

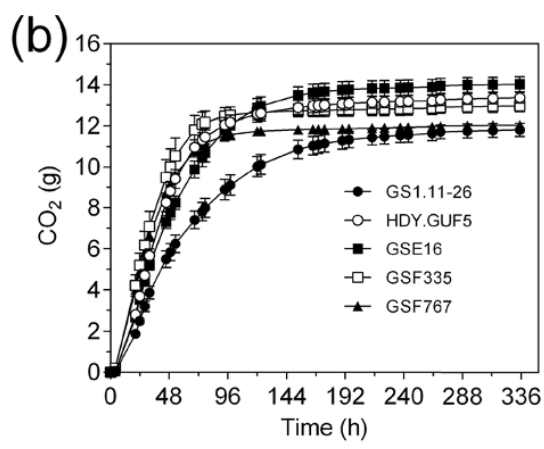

(d)

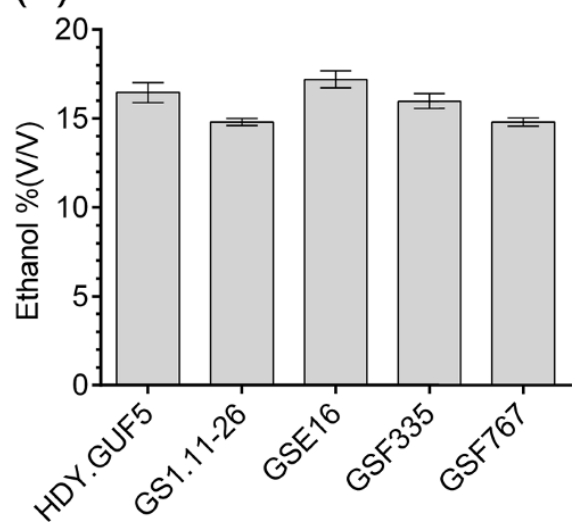

Figure 13 Very-high gravity fermentation in YP with $330 \mathrm{~g} / \mathrm{L}$ glucose. (a) and (b) show the fermentation profile in continuous stirring and static conditions, respectively. The figures in (c) and (d) show the final ethanol titer produced during fermentation of (a) and (b), respectively. Fermentation was started at an initial cell density of $1.3 \mathrm{~g}$ DW/L.

$[24,29,44]$. A strain tolerant to this medium would most likely also be tolerant to inhibitors in other lignocellulosic hydrolysates that are pretreated in a less severe way. Besides, acid based thermochemical pretreatment is considered to be one of the most cost-competitive methods of pretreatment for bioconversion of lignocellulosic feedstocks in liquid biofuel production $[10,45]$. Hence, such strains would be expected to have broad application potential.

Hybridization of industrial strains is among the most effective and simple techniques used for improving and combining various industrially relevant traits [46,47]. In a classical breeding strategy, haploid strains of opposite mating type are crossed to produce new diploid progeny. However, this method cannot be directly applied to industrial strains since most industrial strains are diploid, polyploid or aneuploid. Hence, the identification of a haploid meiotic segregant with in principle the same superior profile of traits as the parent industrial strain is required and this becomes exceedingly difficult when it concerns traits that are only important at industrial scale. Hence, the haploid progeny used for breeding will never display exactly the same repertoire of positive traits as the original diploid strain. This makes breeding of industrial yeast strains into a huge challenge. In our work, we have used for mating the original diploid $M A T \alpha / \alpha$ strain, GS1.11-26, rather than a haploid derivative, so as to maintain the entire genetic basis underlying the superior D-xylose fermentation capacity of that strain. Constructing hybrid strains by mating two mating competent diploid parents has been described previously [48]. However, in that report, no further meiotic recombination step had been applied to the tetraploid hybrid strains to isolate diploid segregants, which might be advantageous for two reasons. First, the most stable genome size in S. cerevisiae appears to be the diploid state. This is suggested by the frequent spontaneous evolution of ploidy from tetraploid to diploid in both optimal and stressful environments [49]. This implies that a tetraploid industrial strain will likely show lower stability and might easily lose important traits when losing chromosomes in the shift to a lower ploidy during its industrial usage. Second, meiotic recombination can generate great diversity in a cell population and sporulation of a tetraploid can therefore generate strains with superior performance compared to the tetraploid parent strain [50]. This was also observed in our work, in which diploid segregants were obtained with a much higher $\mathrm{D}$-xylose utilization rate than the tetraploid parent. This is probably due to 
the involvement of recessive alleles in sustaining efficient D-xylose utilization.

We have used two approaches to improve the GS1.11-26 strain. The backcrossing with an Ethanol Red segregant was mainly aimed at eliminating the negative traits that had been introduced in the background of the strain during its development for high xylose fermentation capacity and inhibitor tolerance, since we maintained the original Ethanol Red background in the breeding process. This approach has been previously described as beneficial for weeding out deleterious mutations in mutant strains [51,52]. On the other hand, breeding with the Fseg25 strain introduced a new, albeit also industrial, genetic background and was mainly aimed at further enhancing inhibitor tolerance, with the risk of losing traits important for a bioethanol production strain. Both approaches were successful in generating strongly improved strains. The GSE16 strain had not only lost the low glucose utilization and aerobic growth rate of its parent, GS1.11-26, but also displayed inhibitor tolerance to a level at least as high as the original unevolved parent strain, HDY.GUF5. The GSF335 and GSF767 strains also largely lost the negative properties of their GS1.11-26 parent, but in addition gained at least part of the very high inhibitor tolerance of the Fseg25 parent strain.

The three hybrid strains were evaluated for various industrially relevant traits, including tolerance to different inhibitors, fermentation performance in a D-xylose/glucose mixture and in very-high gravity fermentation. All three new hybrid strains showed much better general performance in various stress conditions compared to GS1.11-26. There was no single strain that stood out in all conditions. The relative performance varied with the test. In a semi-anaerobic batch fermentation of spruce hydrolysate, the three strains showed a much shorter lag phase compared to GS1.11-26. On the other hand, the very high D-xylose fermentation capacity of GS1.11-26 was not fully maintained in the new hybrid strains. Even though the three hybrid strains were able to consume all the $37 \mathrm{~g} / \mathrm{L}$ D-xylose and $36 \mathrm{~g} / \mathrm{L}$ glucose in $32 \mathrm{~h}$, they retained only $35 \%$ to $60 \%$ of the maximum D-xylose utilization rate of GS1.11-26. The higher inhibitor tolerance of the three new hybrid strains can explain why they displayed D-xylose utilization rates in spruce hydrolysate comparable to that of the strain GS1.11-26. The rate of glucose utilization was also significantly higher than that of GS1.11-26, which is likely also due to their higher tolerance to the inhibitors in spruce hydrolysate. Tolerance to multiple stress factors has previously been shown to correlate with high ethanol yield and a high ethanol production rate in S. cerevisiae [28]. Moreover, D-xylose fermentation is more sensitive to stress factors, especially to acetic acid, compared to glucose fermentation [26]. The severe reduction of tolerance to acetic acid (and possibly to other inhibitors) of GS1.11-26 [27] can explain its lower D-xylose utilization rate in spruce hydrolysate compared to synthetic medium. On the other hand, the rate of D-xylose utilization by the three new hybrid stains was not as severely reduced as for GS1.11-26 in the spruce hydrolysate compared to complex medium, which can be explained by their higher inhibitor tolerance.

Other diploid hybrid segregants with a higher D-xylose fermentation capacity than the three selected strains have been isolated, but they displayed very slow growth rates in glucose and also reduced inhibitor tolerance. Hence, they were excluded because of these negative properties. The difficulty in maintaining the superior D-xylose fermentation rate of the parent strain, GS1.11-26, might suggest that one or more of the negative background mutations in the strain GS1.11-26 might either be causally or structurally linked to the high D-xylose fermentation rate. If these traits are causally linked, the beneficial genes or loci important for efficient D-xylose fermentation might be linked with the reduced growth rate or with the higher inhibitor tolerance. This would mean that it will not be possible or be very difficult to combine high general robustness and high D-xylose utilization capacity in this strain background. However, if the negative mutations are only structurally linked to the positive genetic modifications, i.e. residing close to each other in the genome, they could be removed without affecting the superior D-xylose fermentation performance. Further research, therefore, should focus on the identification of the genetic basis of the efficient D-xylose fermentation as well as the high inhibitor tolerance, so that they can be engineered by reverse metabolic engineering.

Bioethanol production is commonly performed in veryhigh gravity fermentations using highly concentrated substrates so that a maximal final ethanol titer can be reached [53]. These substrates include first-generation feedstocks, such as sugar cane-molasses, starch or grains, and in the future, second-generation feedstocks, in particular lignocellulose waste steams and bioenergy crops. A high final ethanol titer has multiple advantages. It reduces the ethanol distillation costs but also lowers the liquid volumes in the plant causing large savings in heating, cooling, pumping and transport costs. Very-high gravity fermentation causes high stress, in particular osmostress in the beginning of the fermentation and ethanol stress at the end of the fermentation. This results in a longer fermentation time and lower ethanol yield as a result of higher residual sugar [54]. Hence, the performance of a new yeast strain in very-high gravity fermentation is a crucial quality criterion for its use in real industrial practice. This was especially important since the parent strain, GS1.11-26, was severely compromised in maximal ethanol accumulation capacity in very-high gravity fermentations [27]. Hence, we evaluated the three new hybrid strains 
also in very-high gravity fermentation and found that they were not only much better than GS1.11-26, but showed even improved performance in some cases compared to the original HDY.GUF5 strain. The hybrid strain, GSF335, exhibited significantly increased performance compared to its tetraploid parent as well as to both its diploid progenitor strains GS1.11-26 and Fseg25. This clearly shows that meiotic recombination with diploid strains is able to further improve important performance traits that are considered to be already very high in the very best currently used bioethanol production strains.

\section{Conclusions}

We have successfully developed three robust industrial yeast strains that combine efficient D-xylose utilization with high inhibitor tolerance for use in bioethanol production with lignocellulose hydrolysates. Two of the strains (GSF3 35 and GSF767) have been derived through meiotic recombination of the efficient D-xylose utilizing strain GS1.11-26 (derived from Ethanol Red) with the most inhibitor tolerant strain obtained from a screening of more than 580 yeast strains. Strain GSE16 has a purely Ethanol Red background, developed by backcrossing GS1.11-26 with a haploid derivative of Ethanol Red. All three strains showed superior performance with respect to aerobic growth rate, glucose consumption rate and inhibitor tolerance compared to GS1.11-26. The D-xylose utilization rate of the three strains in complex medium was reduced compared to GS1.11-26, but in inhibitor-rich acid-pretreated spruce hydrolysate it was comparable. Due to the high robustness, the proven record in industrial application of the background strain Ethanol Red, and the efficient D-xylose utilization capacity, the three strains have strong potential for direct application in industrial bioethanol production. This study demonstrates that strains with an optimal profile of industrially important traits can be obtained through meiotic recombination directly with diploid strains and by screening large numbers of strains or segregants in a multi-step selection process, using simple high-throughput screens to eliminate poor performers to more elaborate evaluation conditions, mimicking closely the industrial conditions to select the very best performers.

\section{Methods}

\section{Yeast strains and media}

Yeast strains used in this study are listed in Table 1. Yeast cells were propagated in yeast extract peptone (YP) medium $(10 \mathrm{~g} / \mathrm{L}$ yeast extract, $20 \mathrm{~g} / \mathrm{L}$ bacteriological peptone) supplemented with either $20 \mathrm{~g} / \mathrm{L}$ D-xylose (YPX) or $20 \mathrm{~g} / \mathrm{L}$ glucose (YPD). For preparation of solid plates, $15 \mathrm{~g} / \mathrm{L}$ Bacto agar was added after adjusting the $\mathrm{pH}$ to 6.5 with $4 \mathrm{M} \mathrm{KOH}$. Inocula for fermentation and growth tests were prepared by growing strains in YPD medium to stationary phase, harvesting by centrifugation at $2100 \mathrm{~g}$ for $5 \mathrm{~min}$ at $4^{\circ} \mathrm{C}$, and washing with ice-cold sterile Milli-Q water.

\section{Spruce hydrolysate}

The spruce hydrolysate used in this study has been provided by SEKAB E-Technology AB (Örnsköldsvik, Sweden). It was prepared from spruce wood chips by pretreatment with $\mathrm{SO}_{2}$ impregnated steam explosion. The composition of the hydrolysate has been reported previously (20).

\section{Screening of strains for inhibitor tolerance in spruce hydrolysate medium}

Initial screening of the yeast strain collection for inhibitor tolerance was performed on agar plates containing increasing concentrations of the whole slurry of spruce hydrolysate (40\% to $70 \%$ ) supplemented with yeast extract $(10 \mathrm{~g} / \mathrm{L})$ and bacteriological peptone $(20 \mathrm{~g} / \mathrm{L})$. The $\mathrm{pH}$ was adjusted to 5.5 using $4 \mathrm{M} \mathrm{KOH}$. Further screening of the selected strains was performed under semi-anaerobic fermentation conditions using whole slurry of the pretreated spruce material diluted to $30 \%$ in YP medium and supplemented with glucose to a final concentration of $200 \mathrm{~g} / \mathrm{L}$, taking into account the initial glucose concentration already present in the hydrolysate. For screening of segregants from the most tolerant strain JT21653, the concentration of spruce hydrolysate was increased to $77 \%$ and, was supplemented with YP and glucose $70 \mathrm{~g} / \mathrm{L}$ glucose. The fermentations were started at a cell density of $1.3 \mathrm{~g} / \mathrm{L}$, in $60 \mathrm{~mL}$ volume at $30^{\circ} \mathrm{C}$ with continuous stirring at $200 \mathrm{rpm}$. The apparent rate of fermentation was then followed by measuring the weight loss due to $\mathrm{CO}_{2}$ release.

\section{Screening segregants for growth and fermentation in D-xylose medium}

Prescreening of segregants for the ability to grow in D-xylose medium was performed in a 24 well plate containing $1 \mathrm{~mL}$ of YP medium supplemented with $20 \mathrm{~g} / \mathrm{L} \mathrm{D}$-xylose. Cells were inoculated at an initial $\mathrm{OD}_{600}$ of 1.0 from a preculture grown overnight in YPD medium. The $\mathrm{OD}_{600}$ was measured after $20 \mathrm{~h}$ cultivation at $30^{\circ} \mathrm{C}$ in an orbital shaker.

To select strains that were able to ferment D-xylose efficiently, small tube semi-anaerobic batch fermentations were performed at $35^{\circ} \mathrm{C}$ in $50 \mathrm{~mL}$ YP medium containing $40 \mathrm{~g} / \mathrm{L}$ D-xylose as a carbon source. For this purpose, cylindrical tubes were used with a volume of $150 \mathrm{~mL}$ and fitted with a rubber stopper containing cotton-plugged glass tubing. The initial inoculum density was $1.3 \mathrm{~g} \mathrm{DW} / \mathrm{L}$. The cultures were continuously stirred with a magnetic rod at $120 \mathrm{rpm}$. The rate of fermentation was estimated by following the weight loss due to $\mathrm{CO}_{2}$ release. 
Table 1 Yeast strains used in this study, including the 14 strains selected for highest inhibitor tolerance in spruce hydrolysate

\begin{tabular}{|c|c|c|}
\hline Name & Origin & Source/reference \\
\hline JT21585 & Baker's yeast & Puratos, Belgium \\
\hline JT21586 & Baker's yeast & Puratos, Belgium \\
\hline JT21587 & Baker's yeast & Puratos, Belgium \\
\hline JT21621 & Baker's yeast & Anchor Yeast, South Africa \\
\hline JT21651 & Baker's yeast & Algist Bruggeman, Belgium \\
\hline JT21652 & Baker's yeast & Algist Bruggeman, Belgium \\
\hline JT21653 & Baker's yeast & AB Mauri, Australia \\
\hline JT21698 & Distiller's yeast & Lallemand, Canada \\
\hline JT22194 & Beer production yeast & Kasteel triple beer, Belgium \\
\hline JT22231 & Wine yeast & LALVIN (Lallemand, Canada) \\
\hline JT22272 & Natural isolate from apple vinegar & Natural isolate, Slovenia \\
\hline JT22280 & Natural isolate from apple vinegar & Natural isolate, Slovenia \\
\hline JT22728 & Baker's yeast & PYCC-Portuguese Yeast Culture Collection, Portugal \\
\hline FY290 & Natural isolate from fruit & BCCM/MUCL (Mycothèque de I'Université Catholique de Louvain; Belgium) \\
\hline Ethanol Red & Bioethanol production yeast & Fermentis, a division of S. I. Lesaffre, France \\
\hline HDY.GUF5 & $\begin{array}{l}\text { Ethanol Red background, carrying D-xylose and } \\
\text { L-arabinose metabolism gene cassette }\end{array}$ & {$[27]$} \\
\hline GS1.11-26 & HDY.GUF5 background, evolved for D-xylose fermentation & {$[27]$} \\
\hline Fseg25 & Segregant of JT21653 & This study \\
\hline ER17 & Segregant of Ethanol Red & This study \\
\hline GSF335 & Hybrid between GS1.11-26 and Fseg25 & This study \\
\hline GSF767 & Hybrid between GS1.11-26 and Fseg25 & This study \\
\hline GSE16 & Hybrid between GS1.11-26 and ER17 & This study \\
\hline
\end{tabular}

Evaluation of strains for fermentation performance in a D-xylose-glucose mixture

For evaluation of the fermentation performance in medium containing D-xylose and glucose, the batch fermentations were performed in complex medium in $300 \mathrm{~mL}$ shake flasks with a working volume of $200 \mathrm{~mL}$ at $35^{\circ} \mathrm{C}$. The initial inoculum density was $1.3 \mathrm{~g} \mathrm{DW} / \mathrm{L}$. Flasks were closed with fermentation locks containing glycerol. Nitrogen gas was sparged after cell inoculation until the oxygen concentration reached about $2 \mathrm{ppm}$. Cultures were continuously stirred at $120 \mathrm{rpm}$ using a magnetic stirrer. Samples were taken every few hours with needles for analysis.

\section{Evaluation of strains for fermentation performance in spruce medium}

For evaluation of fermentation performance in spruce hydrolysate, the pretreated spruce material was used at a solid loading of $11 \%$ (corresponding to about $50 \%$ of the slurry) in $250 \mathrm{~mL}$ flasks with a working volume of $150 \mathrm{~mL}$. Yeast Nitrogen base $1.7 \mathrm{~g} / \mathrm{L}$ and ammonium sulfate $(5 \mathrm{~g} / \mathrm{L})$ were added as supplement [21]. Since the pretreated spruce material was not enzymatically hydrolyzed, glucose and D-xylose were added to a final concentration of about $62 \mathrm{~g} / \mathrm{L}$ and $18 \mathrm{~g} / \mathrm{L}$. The fermentation was started at an initial cell density of $4 \mathrm{~g}$ DW/L and incubated at $35^{\circ} \mathrm{C}$ with continuous stirring with a magnetic rod at $200 \mathrm{rpm}$. Samples were taken every few hours for analysis through plastic tubing fitted to the bottom side of the flasks, without introducing air.

\section{Very-high gravity fermentation}

Very-high gravity fermentation was performed as previously described [55], in $150 \mathrm{~mL}$ volume cylindrical tubes containing $100 \mathrm{~mL}$ YP medium supplemented with $330 \mathrm{~g} / \mathrm{L}$ glucose at $30^{\circ} \mathrm{C}$. The prepared inoculum was resuspended in about $20-30 \mathrm{~mL}$ aliquot of the medium that was to be used for the fermentation, and inoculated into $100 \mathrm{~mL}$ final volume at an initial cell density of $1.3 \mathrm{~g} \mathrm{DW} / \mathrm{L}$. Agitation was done with a magnetic rod at $120 \mathrm{rpm}$ either continuously or for the first $4 \mathrm{~h}$ (in case of static fermentations). The fermentation rate was followed from the weight loss due to $\mathrm{CO}_{2}$ release. Samples were taken at the end for analysis. 


\section{Determination of ploidy by flow cytometry}

Flow cytometry analysis of DNA content was performed according to the method reported previously [56]. Briefly, exponentially growing cells were washed with ice-cold sterile water and fixed with $70 \%$ ethanol. Cells were treated with RNase $(1 \mathrm{mg} / \mathrm{mL})$ and the DNA was stained with propidium iodide $(0.046 \mathrm{M})$ in $50 \mathrm{mM}$ Tris, $\mathrm{pH} 7.7$ and $15 \mathrm{mM} \mathrm{MgCl} 2$, at $4{ }^{\circ} \mathrm{C}$ for about $48 \mathrm{~h}$. The fluorescence intensity was measured using a FACScan instrument (Becton Dickinson).

\section{Meiotic recombination}

Strains of opposite mating type were crossed by mixing small amounts of cells from each strain on a YPD plate. After $24 \mathrm{~h}$ incubation at $30^{\circ} \mathrm{C}$, the cells were mixed again and re-incubated for another $24 \mathrm{~h}$ to increase the mating efficiency. The mixture was subsequently spread for single colonies on YPD plates. A few colonies were analyzed by PCR and flow cytometry to identify diploids. The selected MATa $/ \alpha$ diploids were sporulated in $1 \%$ potassium acetate for 5 to 7 days, at $23^{\circ} \mathrm{C}$. Spores were isolated by tetrad dissection using an MSM micromanipulator (Singer instruments, Somerset, UK).

\section{Determination of mating type}

Mating type was determined by PCR and pheromone assay. PCR was performed using a primer annealing to the MAT locus and a MATa or MATa specific primer [57]. The mating type was further validated by a pheromone assay. For that purpose, small amounts of cells from two tester strains of S. cerevisiae, MATa bar1- $\triangle$ and MATa sst2- $\Delta$ were inoculated in $1 \%$ agar at $50^{\circ} \mathrm{C}$ in separate tubes. The mixture was immediately poured on top of a YPD plate. After the top agar solidified, about $10 \mu \mathrm{l}$ of cell suspension from the strains to be tested, was spotted onto

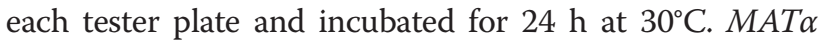
cells showed a zone of growth inhibition on plates of the bar1- $\triangle$ strain while MATa cells showed a zone of growth inhibition on plates of the sst2- $\Delta$ strain. Diploid cells did not produce a zone of inhibition.

\section{Bioscreen growth rate assay}

To perform the growth rate assay, strains were first pregrown to early stationary phase in YPD medium. After washing the pellets in cold sterile water, the cells were inoculated into $200 \mu \mathrm{l}$ synthetic medium containing $20 \mathrm{~g} / \mathrm{L}$ glucose at an initial $\mathrm{OD}_{600}$ value of 0.1 . The titer plates were incubated at $35^{\circ} \mathrm{C}$ with continuous shaking in a Bioscreen $C$ reader (Labsystems) in which the $\mathrm{OD}_{600}$ values were monitored every $30 \mathrm{~min}$.

\section{Analysis of cell mass and metabolite concentrations} The cell dry weight (DW) for inoculation into the fermentation medium was estimated based on the Optical
Density $\left(\mathrm{OD}_{600 \mathrm{~nm}}\right)$. The DW was first measured by filtering a $10 \mathrm{~mL}$ culture aliquot with a known $\mathrm{OD}_{600}$ value over a preweighed $0.2 \mathrm{~mm}$ Supor Membrane disc filter (PALL Corporation, USA), washing the filter with MilliQ water, and drying it in a microwave oven at about 150 watt for $15-20$ min to constant weight. The correlation between dry weight and the $\mathrm{OD}_{600}$ value was determined for each strain tested.

Metabolites and substrates in samples from fermentation experiments were analyzed by Waters Isocratic Breeze HPLC system (Waters, Milford, MA, USA) using ion-exchange column WAT010290 and a refractive index detection system (Waters 2414 RI detector). Column temperature was maintained at $75^{\circ} \mathrm{C}$ and $5 \mathrm{mM} \mathrm{H}_{2} \mathrm{SO}_{4}$ was used as eluent at a flow rate of $1 \mathrm{~mL} / \mathrm{min}$. For analysis of hydrolysate medium, samples were first centrifuged in $15 \mathrm{~mL}$ falcon tubes at $4000 \mathrm{~g}$ for $10 \mathrm{~min}$. The supernatant was further centrifuged in $2 \mathrm{~mL}$ microcentrifuge tubes at 20,000 $\mathrm{g}$ for $5 \mathrm{~min}$ and then filtered using $0.2 \mu \mathrm{m}$ filters. The filtrate was used for HPLC analysis. Ethanol from the very-high gravity fermentations was measured by near infrared spectroscopy (Alcolyzer, Anton Paar). Rates and yields were calculated as previously described [58].

\section{Abbreviations \\ DW: Dry weight; OD: Optical density; YPD: Yeast extract peptone dextrose; YPX: Yeast extract peptone D-xylose; HPLC: High performance liquid chromatography; PCR: Polymerase chain reaction.}

\section{Competing interests}

The authors declare that they have no competing interests.

\section{Authors' contributions}

MMD, FD, MRFM and JMT designed the experiments. MMD, YL and TB performed the experiments and MMD, FD, YL, TB, MRFM and JMT analyzed the results. MMD drafted the manuscript. FD participated in the drafting of the manuscript. MRFM and FD commented on the manuscript and provided academic supervision. JMT led the study and revised the manuscript. All authors have read and approved the final manuscript.

\section{Acknowledgments}

The authors gratefully acknowledge Alessandro Petitti for help with the screening of the strain collection, Jean-Paul Meijnen, Ben Souffriau, Georg Hubmann, Yudi Yang, Stijn De Graeve, and all other members of the Genetic Analysis Group for stimulating discussions, Thomas Talpe and Stephen Castermans for technical support, and Nico Vangoethem for informatics support and help with the preparation of the figures. We are also grateful to Carl-Axel Lalander, Torbjörn van der Meulen and Sune Wännström

(Sekab E-Technology, Örnsköldsvik, Sweden) for kind provision of lignocellulose hydrolysates. This work has been supported by a predoctoral fellowship from the KU Leuven Interfaculty Council for Development Cooperation (IRO) to MMD and from the China Scholarship Council to YL, SBO grant (IWT 90043) from IWT-Flanders, the EC 7th Framework program (NEMO project) and IOF-Knowledge platform (IKP/10/002 ZKC 1836) to JMT.

Received: 10 June 2013 Accepted: 20 August 2013 Published: 26 August 2013

\section{References}

1. Randelli F: An integrated analysis of production costs and net energy balance of biofuels. Reg Environ Chang 2009, 9:221-229.

2. Nevoigt E: Progress in metabolic engineering of Saccharomyces cerevisiae. Microbiol Mol Biol Rev 2008, 72:379-412. 
3. Mielenz JR: Ethanol production from biomass: technology and commercialization status. Curr Opin Microbiol 2001, 4:324-329.

4. Sims RE, Mabee W, Saddler JN, Taylor M: An overview of second generation biofuel technologies. Bioresour Technol 2010, 101:1570-1580.

5. Sanchez OJ, Cardona CA: Trends in biotechnological production of fuel ethanol from different feedstocks. Bioresour Technol 2008, 99:5270-5295.

6. Kim S, Dale BE: Global potential bioethanol production from wasted crops and crop residues. Biomass Bioenergy 2004, 26:361-375.

7. Macrelli S, Mogensen J, Zacchi G: Techno-economic evaluation of 2nd generation bioethanol production from sugar cane bagasse and leaves integrated with the sugar-based ethanol process. Biotechnol Biofuels 2012, 5:22.

8. Schubert C: Can biofuels finally take center stage? Nat Biotechnol 2006, 24:777-784.

9. Weber C, Farwick A, Benisch F, Brat D, Dietz H, Subtil T, Boles E: Trends and challenges in the microbial production of lignocellulosic bioalcohol fuels. Applied Microbiol Biotechnol 2010, 87:1303-1315.

10. Galbe M, Zacchi G: Pretreatment of lignocellulosic materials for efficient bioethanol production. Adv Biochem Eng/Biotechnol 2007, 108:41-65.

11. Taherzadeh MJ, Karimi K: Pretreatment of lignocellulosic wastes to improve ethanol and biogas production: a review. Int J Mol Sci 2008, 9:1621-1651.

12. Jørgensen $H$, Kristensen JB, Felby C: Enzymatic conversion of lignocellulose into fermentable sugars: challenges and opportunities. Biofuels, Bioprod Biorefin 2007, 1:119-134.

13. van Zyl WH, Lynd LR, den Haan R, McBride JE: Consolidated bioprocessing for bioethanol production using Saccharomyces cerevisiae. Adv Biochem Eng/Biotechnol 2007, 108:205-235

14. Hahn-Hagerdal B, Karhumaa K, Fonseca C, Spencer-Martins I, GorwaGrauslund MF: Towards industrial pentose-fermenting yeast strains. Appl Microbiol Biotechnol 2007, 74:937-953.

15. Kuyper M, Hartog MM, Toirkens MJ, Almering MJ, Winkler AA, van Dijken JP, Pronk JT: Metabolic engineering of a xylose-isomerase-expressing Saccharomyces cerevisiae strain for rapid anaerobic xylose fermentation. FEMS Yeast Res 2005, 5:399-409.

16. Wahlbom CF, van Zyl WH, Jonsson LJ, Hahn-Hagerdal B, Otero RR: Generation of the improved recombinant xylose-utilizing Saccharomyces cerevisiae TMB 3400 by random mutagenesis and physiological comparison with Pichia stipitis CBS 6054. FEMS Yeast Res 2003, 3:319-326.

17. Zhou H, Cheng JS, Wang BL, Fink GR, Stephanopoulos G: Xylose isomerase overexpression along with engineering of the pentose phosphate pathway and evolutionary engineering enable rapid xylose utilization and ethanol production by Saccharomyces cerevisiae. Metab Eng 2012, 14:611-622.

18. Brat $D$, Boles $E$, Wiedemann B: Functional expression of a bacterial xylose isomerase in Saccharomyces cerevisiae. Appl Environ Microbiol 2009, 75:2304-2311

19. Bettiga M, Hahn-Hagerdal B, Gorwa-Grauslund MF: Comparing the xylose reductase/xylitol dehydrogenase and xylose isomerase pathways in arabinose and xylose fermenting Saccharomyces cerevisiae strains. Biotechnol Biofuels 2008, 1:16

20. Larsson S, Nilvebrant NO, Jonsson LJ: Effect of overexpression of Saccharomyces cerevisiae Pad1p on the resistance to phenylacrylic acids and lignocellulose hydrolysates under aerobic and oxygen-limited conditions. Appl Microbiol Biotechnol 2001, 57:167-174.

21. Larsson S, Cassland P, Jonsson LJ: Development of a Saccharomyces cerevisiae strain with enhanced resistance to phenolic fermentation inhibitors in lignocellulose hydrolysates by heterologous expression of laccase. Appl Environ Microbiol 2001, 67:1163-1170.

22. Gorsich SW, Dien BS, Nichols NN, Slininger PJ, Liu ZL, Skory CD: Tolerance to furfural-induced stress is associated with pentose phosphate pathway genes ZWF1, GND1, RPE1, and TKL1 in Saccharomyces cerevisiae. Appl Microbiol Biotechnol 2006, 71:339-349.

23. Hou X, Yao S: Improved inhibitor tolerance in xylose-fermenting yeast Spathaspora passalidarum by mutagenesis and protoplast fusion. Appl Microbiol Biotechnol 2012, 93:2591-2601.

24. Koppram R, Albers E, Olsson L: Evolutionary engineering strategies to enhance tolerance of xylose utilizing recombinant yeast to inhibitors derived from spruce biomass. Biotechnol Biofuels 2012, 5:32.

25. Olofsson K, Bertilsson M, Liden G: A short review on SSF - an interesting process option for ethanol production from lignocellulosic feedstocks. Biotechnol Biofuels 2008, 1:7.
26. Bellissimi E, van Dijken JP, Pronk JT, van Maris AJ: Effects of acetic acid on the kinetics of xylose fermentation by an engineered, xylose-isomerase-based Saccharomyces cerevisiae strain. FEMS Yeast Res 2009, 9:358-364.

27. Demeke MM, Dietz H, Li Y, Foulquie-Moreno MR, Mutturi S, Deprez S, Den Abt T, Bonini BM, Liden G, Dumortier F, et al: Development of a D-xylose fermenting and inhibitor tolerant industrial Saccharomyces cerevisiae strain with high performance in lignocellulose hydrolysates using metabolic and evolutionary engineering. Biotechnol Biofuels 2013, 6:89.

28. Zheng DQ, Wu XC, Tao XL, Wang PM, Li P, Chi XQ, Li YD, Yan QF, Zhao YH: Screening and construction of Saccharomyces cerevisiae strains with improved multi-tolerance and bioethanol fermentation performance. Bioresour Technol 2011, 102:3020-3027.

29. Almeida JR, Modig T, Petersson A, Hahn-Hagerdal B, Liden G, Gorwa-Grauslund MF: Increased tolerance and conversion of inhibitors in lignocellulosic hydrolysates by Saccharomyces cerevisiae. J Chem Technol Biotechnol 2007, 82:340-349.

30. Shuai L, Yang Q, Zhu JY, Lu FC, Weimer PJ, Ralph J, Pan XJ: Comparative study of SPORL and dilute-acid pretreatments of spruce for cellulosic ethanol production. Bioresour Technol 2010, 101:3106-3114.

31. St Charles J, Hamilton ML, Petes TD: Meiotic chromosome segregation in triploid strains of Saccharomyces cerevisiae. Genetics 2010, 186:537-550.

32. Ding MZ, Li BZ, Cheng JS, Yuan YJ: Metabolome analysis of differential responses of diploid and haploid yeast to ethanol stress. OMICS 2010, 14:553-561.

33. Schwartz K, Wenger JW, Dunn B, Sherlock G: APJ1 and GRE3 homologs work in concert to allow growth in xylose in a natural Saccharomyces sensu stricto hybrid yeast. Genetics 2012, 191:621-632.

34. Attfield PV: Stress tolerance: the key to effective strains of industrial baker's yeast. Nat Biotechnol 1997, 15:1351-1357.

35. Zhao XQ, Bai FW: Mechanisms of yeast stress tolerance and its manipulation for efficient fuel ethanol production. J Biotechnol 2009, 144:23-30.

36. Borneman AR, Pretorius IS, Chambers PJ: Comparative genomics: a revolutionary tool for wine yeast strain development. Curr Opin Biotech 2013, 24:192-199.

37. Sonderegger M, Jeppsson M, Larsson C, Gorwa-Grauslund MF, Boles E, Olsson L, Spencer-Martins I, Hahn-Hagerdal B, Sauer U: Fermentation performance of engineered and evolved xylose-fermenting Saccharomyces cerevisiae strains. Biotechnol Bioeng 2004, 87:90-98.

38. Sonderegger $M$, Sauer U: Evolutionary engineering of Saccharomyces cerevisiae for anaerobic growth on xylose. Appl Environ Microbiol 2003, 69:1990-1998.

39. Kuyper M, Toirkens MJ, Diderich JA, Winkler AA, van Dijken JP, Pronk JT: Evolutionary engineering of mixed-sugar utilization by a xylose-fermenting Saccharomyces cerevisiae strain. FEMS Yeast Res 2005, 5:925-934.

40. Wisselink HW, Toirkens MJ, Wu Q, Pronk JT, van Maris AJ: Novel evolutionary engineering approach for accelerated utilization of glucose, xylose, and arabinose mixtures by engineered Saccharomyces cerevisiae strains. Appl Environ Microbiol 2009, 75:907-914.

41. Tomas-Pejo E, Ballesteros M, Oliva JM, Olsson L: Adaptation of the xylose fermenting yeast Saccharomyces cerevisiae F12 for improving ethanol production in different fed-batch SSF processes. I Industrial Microbio Biotechnol 2010, 37:1211-1220.

42. Zhang W, Geng A: Improved ethanol production by a xylose-fermenting recombinant yeast strain constructed through a modified genome shuffling method. Biotechnol Biofuels 2012, 5:46.

43. Almeida JR, Karhumaa K, Bengtsson O, Gorwa-Grauslund MF: Screening of Saccharomyces cerevisiae strains with respect to anaerobic growth in non-detoxified lignocellulose hydrolysate. Bioresour Technol 2009, 100:3674-3677.

44. Persson P, Larsson S, Jonsson LJ, Nilvebrant NO, Sivik B, Munteanu F, Thorneby L, Gorton L: Supercritical fluid extraction of a lignocellulosic hydrolysate of spruce for detoxification and to facilitate analysis of inhibitors. Biotechnol Bioeng 2002, 79:694-700.

45. Agbor VB, Cicek N, Sparling R, Berlin A, Levin DB: Biomass pretreatment: fundamentals toward application. Biotechnol Adv 2011, 29:675-685.

46. Pretorius IS, Bauer FF: Meeting the consumer challenge through genetically customized wine-yeast strains. Trends Biotechnol 2002, 20:426-432.

47. Yamada R, Tanaka T, Ogino C, Kondo A: Gene copy number and polyploidy on products formation in yeast. Appl Microbiol Biotechnol 2010, 88:849-857. 
48. Hashimoto S, Aritomi K, Minohara T, Nishizawa Y, Hoshida H, Kashiwagi S, Akada R: Direct mating between diploid sake strains of Saccharomyces cerevisiae. Appl Microbiol Biotechnol 2006, 69:689-696.

49. Gerstein AC, Chun HJ, Grant A, Otto SP: Genomic convergence toward diploidy in Saccharomyces cerevisiae. PLoS Genet 2006, 2:e145.

50. Teunissen A, Dumortier F, Gorwa MF, Bauer J, Tanghe A, Loiez A, Smet P, Van Dijck $P$, Thevelein JM: Isolation and characterization of a freeze-tolerant diploid derivative of an industrial baker's yeast strain and its use in frozen doughs. Appl Environ Microbiol 2002, 68:4780-4787.

51. Marullo P, Mansour C, Dufour M, Albertin W, Sicard D, Bely M, Dubourdieu D: Genetic improvement of thermo-tolerance in wine Saccharomyces cerevisiae strains by a backcross approach. FEMS Yeast Res 2009, 9:1148-1160.

52. Patnaik R: Engineering complex phenotypes in industrial strains. Biotechnol Prog 2008, 24:38-47.

53. Balat $\mathrm{M}$, Balat $\mathrm{H}$ : Recent trends in global production and utilization of bio-ethanol fuel. Applied Energy 2009, 86:2273-2282.

54. Bai FW, Anderson WA, Moo-Young M: Ethanol fermentation technologies from sugar and starch feedstocks. Biotechnol Adv 2008, 26:89-105.

55. Pais TM, Foulquié-Moreno MR, Hubmann G, Duitama J, Swinnen S, Goovaerts A, Yang Y, Dumortier F, Thevelein JM: Comparative polygenic analysis of maximal ethanol accumulation capacity and tolerance to high ethanol levels of cell proliferation in yeast. PLoS Genet 2013, 9(6):e1003548.

56. Popolo L, Vanoni M, Alberghina L: Control of the yeast cell cycle by protein synthesis. Exp Cell Res 1982, 142:69-78.

57. Huxley C, Green ED, Dunham I: Rapid assessment of $S$. cerevisiae mating type by PCR. Trends Genet 1990, 6:236.

58. Zaldivar J, Roca C, Le Foll C, Hahn-Hagerdal B, Olsson L: Ethanolic fermentation of acid pre-treated starch industry effluents by recombinant Saccharomyces cerevisiae strains. Bioresour Technol 2005, 96:1670-1676.

doi:10.1186/1754-6834-6-120

Cite this article as: Demeke et al:: Combining inhibitor tolerance and Dxylose fermentation in industrial Saccharomyces cerevisiae for efficient lignocellulose-based bioethanol production. Biotechnology for Biofuels 2013 6:120.

\section{Submit your next manuscript to BioMed Central and take full advantage of:}

- Convenient online submission

- Thorough peer review

- No space constraints or color figure charges

- Immediate publication on acceptance

- Inclusion in PubMed, CAS, Scopus and Google Scholar

- Research which is freely available for redistribution 